\title{
La investigación en Chile sobre imaginarios y representaciones sociales
}

\author{
RESEARCH IN CHILE ON IMAGINARIES AND SOCIAL REPRESENTATIONS
}

Rubén Dittus (ruben.dittus@ucentral.cl) Facultad de Comunicaciones, Universidad Central de Chile (Santiago, Chile) ORCID: 0000-0002-7613-1643

Oscar Basulto (oscarbasultogallegos@gmail.com) Facultad de Educación, Universidad Católica de la Santísima Concepción (Concepción, Chile) ORCID: 0000-0001-8323-1098

Ignacio Riffo (ignacioriffopavon@gmail.com) Facultad de Ciencias de la Comunicación, Universidad Autónoma de Barcelona (Barcelona, España) ORCID: 0000-0001-6691-3572

\begin{abstract}
This paper addresses the state of the art that draws on the theory of imagery and social representations in Chile. It is a cartographic study, and as such, it considers those approaches, methodologies and results that are more relevant, which allow a "state of the research" to be sketched out. It is not, therefore, an exhaustive signing of each paper or thesis to which it can be linked with the field indicated, due to the large volume of products associated directly or indirectly with the so-called "social imagery". Semistructured questionnaires were applied to researchers in the area, research progress was reviewed and the most cited scientific articles were analyzed, allowing a content analysis to be applied. It was observed that the authors and theories with which they work are diverse and propose a conceptual crossing that extends to the methodological plane. It is concluded that imagery and social representations have become a good tool to understand reality and that facilitates research questions about the past and present of the relevant and the everyday.
\end{abstract}

Key words: social imaginary, representations, Chile, research, methodology.

\section{Resumen}

Este texto aborda el estado de aquellas investigaciones que se nutren de la teoría de imaginarios y representaciones sociales en Chile. Se trata de un estudio cartográfico, y como tal, toma en consideración aquellos enfoques, metodologías y resultados más relevantes, que permiten bosquejar un "estado de la cuestión". No es, por lo tanto, un fichaje exhaustivo de cada trabajo o tesis al que se pueda vincular con el campo señalado, debido al gran volumen de productos asociados directa o indirectamente con la denominada "imaginería social". Se aplicaron cuestionarios semi-estructurados a investigadores del área, se revisaron avances de investigación y se analizaron los artículos científicos más citados, que permitieron aplicar un análisis de contenido. Se observó que los autores y teorías con las que se trabajan son diversos y proponen una transversalidad conceptual que se extiende al plano metodológico. Se concluye que los imaginarios y representaciones sociales se han convertido en una buena herramienta para comprender la realidad y que facilita preguntas de investigación sobre el pasado y el presente de lo relevante y lo cotidiano. 
Palabras clave: imaginario social, representaciones, Chile, investigación, metodología.

\section{Introducción}

En Chile la teoría de imaginarios y representaciones sociales está anclada en las ciencias sociales. Si bien se trata de un campo de estudio reciente -al igual que en el resto de América Latina- se observa gran influencia epistémica de autores foráneos y de necesidades metodológicas que se ajustan a escuelas y corrientes ya instaladas. Las fronteras se encuentran desdibujadas, debido al diálogo constante con la filosofía y los análisis del discurso, situación que define nuevos horizontes y potencia el necesario pacto interdisciplinar. Al no existir un estado del arte de tal envergadura, y conscientes en la necesidad de que cualquier sistematización epistemológica ayuda a nuevos investigadores, el equipo de autores cree necesario revisar y cimentar los avances investigativos realizados por otros, aclarar rumbos, contrastar conclusiones provisionales y explorar nuevas perspectivas en el campo. Los investigadores escogidos alimentan el panorama que nos interesa dibujar, privilegiando aquellos cuyos estudios y/o reflexiones han tenido cierta repercusión bibliográfica o que cuenten con patrocinios institucionales.

Hemos dividido el texto en tres apartados: enfoque socio-fenomenológico de imaginarios urbanos, enfoque socio-antropológico y enfoque comunicológico. Cada uno de ellos busca sistematizar aquellas corrientes e investigaciones más representativas de este campo en Chile. A lo largo del artículo, el lector observará que la revisión del concepto de lo imaginario se circunscribe indistintamente a cada enfoque o escuela teórica, situación que -lejos de ser un obstáculo- constituyó un desafío para el equipo revisor.

\section{El enfoque socio-fenomenológico de imaginarios urbanos}

Los constantes ejercicios de teoría y práctica propuestos por los trabajos de Manuel Antonio Baeza -y en un vínculo permanente con la filosofía- dan cuenta de una gran preocupación por las dinámicas micro y macro sociales en los diferentes niveles de realidad, pero siempre dando centralidad al concepto de imaginario social. El término es abordado por el sociólogo chileno en su abundante producción bibliográfica, trabajo reflexivo que lo convierte en uno de los teóricos iberoamericanos más importantes del campo y en cita obligada cada vez que se aborda el concepto en lengua española. Fruto de esa predilección es el importante número de tesis de licenciatura y magíster dirigidas que se han acoplado a dichos intereses. Al momento de publicar este artículo dirige el Grupo Concepción de Estudios sobre Imaginarios Sociales, heredero del antiguo GCEIS, con sede en Concepción, vinculado alguna vez al grupo de estudios que encabezó Juan Luis Pintos, en España.

Baeza reivindica el valor de lo simbólico, el papel de la imaginación, la experiencia subjetiva y la importancia de la "facultad instituyente o socialmente validante" (Baeza 2008:100), sustentado en aportes de diferentes disciplinas y autores que van erigiendo la teoría de los imaginarios sociales como una subdisciplina de corriente principal. Los procesos intersubjetivos que fundan los imaginarios sociales tienen mucho que decir y este es uno de los motivos de por qué sería necesaria una "sociología profunda" que aborde la imaginería social. Así, para Baeza, los imaginarios sociales serían "múltiples y variadas construcciones mentales (ideaciones) socialmente compartidas de significancia práctica en el mundo, en sentido amplio, destinadas al otorgamiento de sentido existencial" (Baeza 2003:20). Se trata de una rehabilitación de la subjetividad humana, que pone como eje principal de construcción lo que se entiende por realidad social. El sujeto significante puede ser individual o colectivo, y ambos tipos operan en un sistema de significatividades que son múltiples. Se trata de una contribución fuertemente impregnada de la obra de Cornelius Castoriadis, quien plantea como cuestión fundamental el hecho de que aquello que 
denominamos de manera bastante sucinta "la realidad social" es, finalmente, una construcción social como lo afirmaron para la sociología Peter Berger y Thomas Luckmann. No sería sino el resultado de procesos subjetivos significativos de legitimación de lo que parece ser convertido en lo que es, objetivamente.

La relación entre ideología e imaginarios sociales nutre los análisis del enfoque socio-fenomenológico. Dicha relación operaría en doble sentido, ya sea para configurar producciones imaginarias o para articular sistemas totalizadores de sentido, tal como fue el caso de la Alemania nazi respecto de formas mitologizadas provenientes de ancestrales imaginarios vinculados a la pertenencia germánica. Para este enfoque, el aprovechamiento ideológico es completo cuando su contenido se encuentra homologado por la sociedad respectiva, que la entiende y acepta como lo verdadero. En Chile, el caso del indígena constituye un caso emblemático de discriminación étnica y social, por consolidación de sucesivos imaginarios sociales dominantes en la sociedad chilena. Los trabajos de Manuel Antonio Baeza y Andrea Aravena proponen que el estereotipo se construye socialmente siempre en desconocimiento total o parcial de un grupo social. En el caso estudiado, se da cuenta de la ausencia de voluntad de integración social y que históricamente animó a las sucesivas fracciones de la élite mestiza o criolla en el poder. Desde la perspectiva de la teoría fenomenológica de los imaginarios sociales es posible establecer en este caso la plena correspondencia entre ideología y construcción socio-imaginaria de realidad social. Así, la imagen negativa del indígena en una sociedad heredada del criollismo decimonónico pasó a formar parte de la doxa, como si la fisonomía misma del "indio" quedara plasmada a través de una serie de atributos subjetivamente señalados. Baeza lo explica señalando que "esta violencia simbólica expresa a nivel de Estado el triunfo categórico -aunque jamás definitivo- de una cierta visión de mundo sobre otras también presentes en la sociedad. El Estado cristaliza a nivel máximo los contenidos imaginarios sociales victoriosos, pero desde allí los destila, naturalizándolos hacia la sociedad" (Baeza 2016:204).

En el campo de las investigaciones de alto impacto, destacan los proyectos de Manuel Antonio Baeza, como investigador principal: "Imaginarios sociales del otro en el Chile contemporáneo. La mujer, el indígena y el inmigrante" (FONDECYT N 1071090, ejecutado el período 2007-209), "Chile hoy: vivencias de alteridades e imaginarios sociales de víctimas de violencia simbólica" (FONDECYT $N^{\circ} 1100928$, ejecutado el período 2010-2012), "Construcción imaginaria social de la desconfianza y su relación con el descontento en el Chile actual" (FONDECYT N 1130738, ejecutado el período 2013-2015). Los resultados de esas investigaciones $u$ otros trabajos anteriores han dado lugar a publicaciones indexadas, entre las cuales destacan: "Imaginarios sociales del otro: el personaje del forastero en Chile (de 1845 a nuestros días)" (Silva y Baeza, en Sociedad Hoy 17, pp. 29-38), "Jóvenes chilenos y construcción socio-imaginaria del ser-otro mujer" (Aravena y Baeza, en Última Década 32, pp. 159-171), "Violencia simbólica en el Chile contemporáneo. Estrategias de respuesta en relaciones de alteridad" (Aravena y Baeza, en Revista Internacional de Sociología 71(3), pp. 543-565), "La desconfianza y el descontento en el Chile de la postdictadura. Formas modernas de construcción socio-imaginaria de relaciones sociales" (Aravena y Baeza, en Cinta de Moebio 53, pp. 147-157).

Uno de los resultados que arroja el estudio sobre el cómo los chilenos construyen socialmente la alteridad del Ser-Otro mujer, es el consabido machismo que forma parte de la identidad chilena, entendida como una construcción socio-imaginaria. Para los investigadores Baeza y Aravena, producto de este acto identitario fundacional de violencia simbólica, nuestra cultura tradicional finalmente da cuenta de esta aseveración. En el caso específico del Ser-Otro Mujer, "se concluye que la identidad chilena tiene, básicamente, una característica masculina inserta en nuestros patrones culturales y que la lógica de 
tratamiento masculino inferiorizante de la Otredad femenina se organiza en torno a un binomio 'dentro' (espacio doméstico y privado) / 'fuera' (espacio no doméstico y público)" (Baeza y Aravena 2010:168).

En la misma línea investigativa, la antropóloga Andrea Aravena escribe sobre los problemas generados por la discriminación asociados a los imaginarios del inmigrante y el joven en textos de gran rigor metodológico. Es el caso de "Juventud, migración y discriminación en el Chile contemporáneo" (en Última Década 36, pp. 127-140), artículo que aborda la percepción desde "lo juvenil" de la alteridad en Chile, tanto desde el punto de vista de la población como desde el punto de vista de los inmigrantes a partir de fuentes de información cuantitativas, como el Censo del año 2002 y la encuesta Casen 2006, específicamente las versiones Quinta y Sexta de la serie de Encuestas de Juventud de Chile (2007 y 2009), y de un conjunto de antecedentes cualitativos de producción propia, como resultados de grupos de discusión realizados a inmigrantes jóvenes, resultados de entrevistas en profundidad a inmigrantes peruanos y de grupos de discusión con personas chilenas sobre inmigrantes. El otro texto titulado "Los mapuches más allá y más acá de la frontera: identidad étnica en las ciudades de Concepción y Temuco" (Aravena, Gissi y Toledo, en Sociedad Hoy 8/9, pp. 117-132), se concluye que la identidad que puede o no generar problemas en el seno de la comunidad se transforma en un conflicto identitario cuando se enfrenta a la experiencia de la discriminación y en la diferenciación en la escuela secundaria, en la universidad o en el trabajo en la vida urbana.

Discípulo de Baeza es el sociólogo Juan Pablo Paredes, investigador de la Universidad Diego Portales y miembro del Núcleo de Investigación en Imaginarios Sociales de la Universidad de Chile. Sus temas de interés apuntan a la democratización, la sociedad civil y ciudadanía, la democracia participativa, los movimientos sociales e identidades sociopolíticas; los cuales se alimentan directa o indirectamente por la teoría de imaginarios sociales y los procesos de significación en los sistemas democráticos. Las influencias teóricas de Paredes van asociadas al concepto de la democracia y la disputa por sus significados, los procesos de naturalización del orden social y política de tal orden o las diferencias entre el momento instituyente de lo político y la conformación de la política como una esfera instituida en el orden social y los nuevos focos de control y conflicto social. En el plano de la construcción de subjetividades, Paredes se apoya teóricamente en Zemelman y De la Garza. El primero distingue los procesos constituyentes del orden social, y para quien la conformación de subjetividades colectivas está dada por las capacidades de articulación colectiva entre singularidades en relación a su cotidianeidad y contexto cultural. En tanto, De la Garza introduce el concepto de configuración subjetiva en relación a la posibilidad de reconocer los niveles de análisis de la subjetividad. Ello implica cierta capacidad de "resignificar sentidos culturales, que se van actualizando a medida que es necesario resignificarlos, a la vez que estos nuevos significados pueden volverse sobre las mismas capacidades de significación de las subjetividades" (De la Garza 2001:112). Mención especial merece su artículo publicado en Imagonautas (Número 2, pp. 36-56), donde el autor analiza la figura de los movimientos sociales y su constitución cultural, social y subjetiva, expresada en formas de acción colectiva, que dan cuenta de los procesos de construcción y disputa actuales por la significación del orden sociopolítico.

También desde una línea fenomenológica, pero apoyado por algunos rasgos constructivista-sistémicos, Oscar Basulto plantea su trabajo investigativo desde el estudio y aplicación de los imaginarios sociales en torno a la construcción de realidad intencionada, a partir de un enfoque multidisciplinar amparado en la teoría sociológica, en la comunicología y en la planificación estratégica. En el artículo "Construcción de valor territorial en el imaginario urbano" (en Revista de Investigaciones Políticas y Sociológicas 2(12), pp. 115-126), se rescatan las nociones del uso del espacio público y una profundización en la teoría de imaginarios sociales. Al mismo tiempo, se analiza la necesidad de contar con una ciudadanía más activa, 
cohesionada y comprometida con los retos de su localidad, otorgando relevancia a la definición en un territorio. En suma, se busca ir resignificando los procesos sociales y sus imaginarios socio-urbanos. Basulto, en co-autoría con el Felipe Aliaga Sáez y Julio Cabrera Varela, publica "El grupo de discusión: Elementos para la investigación en torno a los Imaginarios Sociales" (en Prisma Social 9, pp. 136-175), artículo en el que se vincula la investigación cualitativa, la teoría de imaginarios sociales y la técnica del grupo de discusión. En "Relevancia de las NTIC'S en el turismo cultural/museos: perspectiva hermenéutica en el mundo contemporáneo" (Basulto y Taboada de Zúñiga, en Pasos. Revista de Turismo y Patrimonio Cultural 4(12), pp. 859-874), se analizan las interacciones intersubjetivas, a través de las nuevas tecnologías, redes sociales y plataformas virtuales, incorporando la relevancia que pueden suscitar también- los imaginarios desde la perspectiva virtual. Si bien el artículo mencionado es teóricodocumental, es importante señalar que el análisis expuesto se enriquece con un estudio anterior en el cual -utilizando la metodología del benchmarking y de la socio-semiótica- se analizan diversos sitios web de los museos más importantes del mundo y su interacción con los públicos. Estudios posteriores refuerzan los hallazgos reseñados, pero aplicados al imaginario de la juventud. Es el caso del capítulo "Reacciones sociales e invisibilidad mediática frente al imaginario del joven problema" (en Basulto, O. y Aliaga, F. Diálogos sobre juventud en Iberoamérica, Universidad Santiago de Compostela).

\section{El enfoque socio-antropológico}

Los estudios antropológicos revisados citan variados autores y escuelas. Es el caso de los trabajos de María Belén Ortega, investigadora consultada para esta cartografía, quien dialoga con Claude Levi-Strauss y Victor Turner, autores conectados al rito y la performatividad, enfoques teóricos desde donde se llega a John Austin. Desde el intercambio de símbolos y signos la investigadora se conecta con la semiótica desde Ferdinand de Saussure a Sapir, Fernández, Nadel, Singer o Barthes. Similar influencia se observa de estudios culturales con Stuart Hall y el postestructuralismo francés con Foucault, Bourdieu, Balandier y Ricoeur con su praxis y utopía. Lo mismo ocurre como otros latinoamericanos como Grossberg y Briones que integran las escuelas sociocríticas con los estudios culturales desde el sur. Se observan guiños al paradigma de la complejidad y a Carlos Reynoso, así como a algunos de los planteamientos vinculados a Guattari y Deleuze. Otras fuentes consultadas indican que entre los autores más especializados en el estudio del imaginario, se citan con frecuencia a Denise Jodelet (memoria de los lugares), Alain Pessin (imaginario político, imaginario utópico), François Laplantine (mestizajes culturales, medicinas paralelas), Charles Amouroux (institución hospitalaria, nieve, ski), Catherine Dutheil-Pessin (canción, voz), Yves Chalas (imaginario político, imaginarios urbanos), Jean-Paul Bozonnet (imaginario político, naturaleza, montaña), Pierre Le Quéau (semióforos, sociología relacional). En lo específico, algunos trabajos doctorales de este grupo de investigadores abordan el imaginario utópico (Desroche) y la sociología del arte (Bastide, Duvignaud). Según Ortega, en la línea del perspectivismo de-colonial hay influencia de autores como Appadurai, De la Cadena, Blasser, Viveiros de Castro y el boliviano Prada, así como Dussel y Zibechi, en su articulación con la praxis latinoamericana emancipadora y la filosofía política. Este tema se observa en algunos trabajos de Belén Ortega en el área del Trabajo Social, que actualmente desarrolla desde la sociología de las ausencias de Buaventura Sousa Santos, que se traduce a una etnografía de las ausencias, en base a prácticas, significados e imaginarios invisibilizados por las hegemonías (epistémica, sociocultural, generacional). Ello se aborda desde perspectivas constructivistas/deconstructivistas que buscan imaginarios de lo posible, de lo pensable, para tener una visión integral para la investigación y la acción.

Según Ortega, las representaciones, aunque muy fuertemente ligadas a los dispositivos de poder, son frecuentemente diferenciadas entre representaciones simbólicas (ligado a significados en sistemas de convivencia) y representaciones sociales (ligado a significados en estructuras sociales), aunque ambas 
estén también conectadas. En ese sentido, observamos que la decisión sobre el uso de los conceptos de imaginario o de representaciones está ligado a la formación disciplinar del investigador o a las escuelas de pensamiento en las que han estado vinculados, más que a la efectividad de los términos en sí mismos. De ese modo, el uso del término "imaginario" parece tener un mayor sustento en la socioantropología a diferencia de la noción "representaciones sociales", término que se asocia mayormente a los abordajes psico-sociales de este enfoque teórico. En cambio, los trabajos ligados a la construcción de la memoria colectiva en las sociedades son más cercanos al enfoque teórico de las representaciones sociales. En lo que respecta a la sociología del arte, la dimensión imaginaria de las obras y de los fenómenos artísticos se nutre de autores como Pessin, Dutheil-Pessin, Bastide o Duvignaud.

En el enfoque antropológico, se observa preferentemente el trabajo etnográfico, donde el registro se elabora a partir de pautas de observación y análisis en base a marcadores simbólicos y el uso del método biográfico basado en entrevistas en profundidad sobre el período investigado. Esto es refrendado por Ortega, quien sostiene que el trabajo de investigación más individualizado y grupal, se utilizan técnicas de Gestalt y de psicodrama. En tanto, los análisis multidimensionales se nutren de técnicas que provienen del análisis del discurso y el análisis en base a marcadores simbólicos. El caso de la investigadora Marisol Facuse es destacable por las nuevas propuestas teóricas que inserta en Chile, quien coordina desde el año 2010 el Núcleo de Investigación en Imaginarios (junto a los investigadores Juan Pablo Paredes, Nelson Ruminot y Felipe Aliaga) y el Núcleo de Sociología de Arte y las Prácticas Culturales, ambos dependientes de la Facultad de Ciencias Sociales de la Universidad de Chile. El primer grupo se consagra a analizar el concepto de imaginario a partir del trabajo de distintos autores (Castoriadis, Bachelard, Durand, Pessin, Maffesoli, Pintos, Carretero) y horizontes epistemológicos (socio-antropología, teoría de sistemas, psicoanálisis, marxismo) a fin de problematizar esta noción y proponer perspectivas de investigación en distintos ámbitos (acción colectiva, identidades culturales, urbanismo, artes, género, generaciones, etc.). En tanto el segundo núcleo privilegia una aproximación al fenómeno artístico desde las ciencias sociales, buscando desde sus inicios incorporar perspectivas interdisciplinarias a través del diálogo con dominios tales como la musicología, los estudios teatrales, artes visuales, estudios del patrimonio, urbanismo y ciencias de la comunicación.

En el marco del Proyecto FONDECYT N¹000376, titulado "Representación discursiva del cuerpo (salud/enfermedad) en la medicina popular chilena", se genera el artículo "La construcción discursiva de los imaginarios sociales: el caso de la medicina popular chilena" (Álvarez, Álvarez y Facuse, en Onomazein 7, pp. 145-160). En dicho estudio, los investigadores chilenos Gerardo Álvarez, Andrea Álvarez y Marisol Facuse, generan una construcción antropológica-lingüística e imaginaria de la noción de enfermedad, a partir del relato de sanadores portadores de medicina popular en la región del Bío-Bío. Esta investigación indaga sobre la necesidad de comprender el imaginario, desde la cotidianidad o desde el hacer y decir de quienes construyen y son portadores de imaginarios, como es el caso de imaginarios cotidianos universales como salud, enfermedad, cuerpo, vida y muerte, comprendiendo el modo en que se organiza el pensar y obrar de individuos miembros de diferentes culturas en Chile. En términos metodológicos estamos frente a un estudio interdisciplinario (lingüística, psicología, sociología) sobre las representaciones del cuerpo (salud/enfermedad) en la medicina popular chilena.

En el año 2010, Marisol Facuse -desde la vereda de la sociología del arte- aborda la relevancia que puede tener el concepto de utopía como género de expresión literaria, lo cual queda plasmado en su artículo "La utopía y sus figuras en el imaginario social" (en Sociológica 25(72), pp. 201-213). Dicho trabajo encuentra su punto de partida en la tesis doctoral de la autora: "Utopies sur scène: le monde de l'œuvre de la Compagnie Jolie Môme". En 2011, el trabajo de Facuse titulado "Poesía popular chilena: imaginarios y 
mestizajes culturales" (en Atenea 504, pp. 41-53) señala que las manifestaciones artístico-culturales constituyen aspectos fundamentales de nuestros modos de vida en sociedad, ya sea urbana o rural. En este contexto investigativo, se aborda el concepto de mundo arte, las emociones y las significaciones compartidas que redirigen hacia un imaginario común, un imaginario sonoro a través de la verbalización y canto de la poesía, por lo tanto, cobran relevancia instrumentos musicales de acompañamiento y se habla, entonces, de un canto a lo poeta.

En antropología cultural y estudios étnicos destacan los proyectos en que participa Nicolás Gissi, académico de la Universidad de Chile, cuyas líneas de investigación cruzan temáticas como pueblos indígenas y etnicidad, antropología económica y latinoamericana, exclusión e integración social, así como inmigración latinoamericana. Es el caso del Proyecto FONDECYT №1120795 (en el que participa como coinvestigador) titulado "Etnografía de la colonización y recolonización en los territorios de frontera de Chiloé continental. Actores, intercambios y conflictos", así como su Proyecto FONDECYT de Iniciación №11130287 titulado "Migración y procesos de integración y exclusión social de colombianos y mexicanos en Chile. Estudio comparativo de dos casos de movilidad intralatinoamericana".

En el ámbito de la investigación acción con metodologías participativas, las investigadoras María Belén Ortega y Verónica Gómez propone rescatar las vivencias de los que fueron niños y niñas víctimas de explotación sexual y participantes del Proyecto Aura, a través de la reconstrucción de sus trayectorias de vida en el estudio (2015-2017) "Trayectorias, sentidos y sentimientos: una reconstrucción desde quienes fueron niñas, niños y adolescentes víctimas de explotación sexual". En la misma línea fue publicado el trabajo titulado "Experiencias metodológicas de investigación e intervención con niños, niñas y adolescentes" (Ortega, N.B. y Gómez, V. en Osorio, F. Políticas Públicas y Protección de la Infancia en América Latina, vol. 3, Santiago: FACSO). Similar marco teórico se observa en el artículo "Trabajo social como transdisciplina: hacia una teoría de la intervención" (en Cinta de Moebio 54, pp. 278-289), en el cual se desarrollan tres desafíos para la intervención y la investigación en trabajo social y creación de epistemología; y en el trabajo "El cuestionamiento del poder como dominio: repensar los análisis del poder en la acción social" (en Tabula Rasa 22, pp. 323-334), en el que se reflexiona desde el imaginario del poder. Por otro lado, en el artículo "Sistemas, tridimensionalidad y deconstrucción: aplicando complejidad a la investigación social antropológica" (en Revista MAD 28, pp. 61-60) se propone una forma de abordaje de la investigación antropológica que incluya la dimensión simbólica.

También en la línea de los imaginarios sociales y el trabajo social, la investigadora Leticia Arancibia dirige la investigación FONDECYT № 11110328 "Imaginarios sociales del conflicto. Los sistemas de justificación del acuerdo y el disenso en las relaciones entre los actores de enseñanza media". Dichos resultados, entre otros, se sistematizan en un escrito denominado "Conflicto en la escuela. Institución de la democracia y producción de sistemas de justificación de las relaciones entre los actores de la enseñanza media en Chile". El estudio describe y analiza la forma en que profesores y estudiantes de establecimientos de enseñanza media en Chile, justifican el acuerdo y el conflicto en sus relaciones en la escuela, distinguiendo los imaginarios sociales sobre el conflicto dentro del marco de la convivencia escolar y el contexto cultural y socio-político chileno. La investigación se realizó a través de una metodología cualitativa, de corte interpretativo, semi-inductiva y secuencial, a través del muestreo teórico en continua interpelación entre el análisis y la producción de datos, en diferentes establecimientos educacionales primarios en Chile (Valparaíso, Viña del Mar y Santiago), donde se hicieron entrevistas con profesores/as y grupos focales con estudiantes. En el mismo estudio, se analiza los elementos del discurso de los estudiantes en una discusión en torno al sentido de la democracia, desde la perspectiva de Castoriadis. Lo que se observó fue la construcción de un conjunto de significaciones sostenedoras del sistema social dominante, en un 
permanente estado de tensión por parte de los jóvenes, en torno a cómo sostienen sus formas de vida en el contexto capitalista, o buscan alternativas mentales, afectivas e ideológicas alternativas, inclinados hacia el derrumbe del paradigma neoliberal. En otro artículo de las investigadoras Leticia Arancibia, Pamela Soto y Andrea González, titulado "Imaginarios sociales y biopolítica en la escuela: la mujer como cuerpo del delito" (en Cinta de Moebio 55, pp. 29-46), se abordaron las implicancias de los contenidos en disputa en el proceso de subjetivación y construcción de la política, considerando la co-construcción de rol y visión que existe de la mujer en la sociedad occidental y específicamente en la chilena. Se realiza un análisis desde la filosofía clásica hasta la contemporánea. A partir de diversos conceptos se establece el cuerpo femenino como cuerpo del delito, es decir, como componente de una acusación elaborada en el contexto del Estado patriarcal.

\section{Enfoque comunicológico y estudios mediales}

El estudio de la comunicación y la reflexión sobre las representaciones sociales parecen haber nacido juntas. Ello se desprende cada vez que se buscan elementos diferenciadores entre un campo u otro. La condición de transdisciplina hace de la teoría de la comunicación un campo rico en vínculos con la semiótica, la psicología social, el psicoanálisis, la antropología cultural o la sociología profunda, por mencionar solo a algunas barreras epistemológicas. En Chile, los trabajos del enfoque comunicológico se nutren de las teorías del chileno Manuel Antonio Baeza y la escuela francesa, principalmente con los trabajos de Gilbert Durand y, en menor medida, Cornelius Castoriadis y Serge Moscovici. Tienen su predilección por el estudio de las representaciones, estableciendo un énfasis en cómo se (re)presenta la realidad. De esta manera, aborda en un comienzo la batería teórica que ofrecen los imaginarios sociales para luego conectar con la conceptualización de la construcción de la realidad, trabajando sobre la idea de Berger y Luckmann. Se observa que el interés por investigar en torno esta temática nace de la idea de la 'influencia de la prensa en la sociedad', más precisamente en la contribución de la prensa en la construcción de realidades.

La herramienta metodológica preferente es el Análisis Crítico del Discurso, desde la teoría base de Teun van Dijk. Así, por ejemplo, se observa en los trabajos de Rodrigo Browne desde el enfoque de la 'diferencia', centrándose en mapuches, peruanos y bolivianos. Conocidos son los análisis críticos de la construcción social de la realidad a través de la representación mapuche y peruano-boliviano en las noticias de la prensa diaria de cobertura nacional. Asimismo, sus investigaciones se han desarrollado en relación al análisis crítico y complejo del discurso periodístico verbo-visual de la representación intercultural limítrofe/liminal en la prensa boliviana, chilena y peruana sobre el diferendo marítimo internacional de La Haya. A su vez, los trabajos de Alberto Mayorga y Luis Nitrihual también se suman a esta corriente teórica. En sus contribuciones se pueden observar el interés por evidenciar empíricamente los sistemas de significación que sustentan las construcciones socioimaginarias que operan en la esfera pública, mediante la producción discursiva que emerge desde diversos dispositivos sociales, políticos, económicos y culturales.

En el marco del proyecto FONDECYT № 11070062, titulado “Comunicación intercultural y periodismo intercultural: análisis crítico de la construcción social de la realidad través de la representación mapuche y peruano-boliviana en las noticias de la prensa diaria de cobertura nacional (Copesa y El Mercurio)", Rodrigo Browne junto a Víctor Silva y Ricardo Baessolo, publicaron "Periodismo intercultural: representación peruana y boliviana en la prensa chilena" (en Revista Científica Educomunicación: Comunicar 35(18), pp. 85-93). El objetivo de este artículo es comprender cómo son representados los «discursos de la diferencia» de lo peruano y boliviano en Chile. Dentro de este mismo proyecto FONDECYT 
se desprende el artículo titulado: "Análisis crítico del discurso de la representación boliviana en las noticias de la prensa diaria de cobertura nacional: el caso de El Mercurio y La Tercera" (Browne y Romero, en Revista Polis 9(26), pp. 1-17). En esta investigación sus autores pretenden descubrir cómo la prensa escrita colabora en la representación y construcción de la realidad intercultural de sus lectores, formando y promoviendo prejuicios y estereotipos socioculturales. A su vez, los autores ponen en cuestión el concepto de 'objetividad' en la práctica del periodismo, así como la conceptualización de 'realidad'.

Por su parte, desde la matriz del proyecto FONDECYT (№ 11070264) "Periodismo intercultural: construcción de la noticia a través de un análisis crítico y complejo del discurso en la prensa diaria de cobertura nacional de Chile y Perú. El caso de El Mercurio y La Cuarta; y El Comercio y Ajá", Browne junto a otros investigadores publican los artículos "Informar al otro, una mirada desde el periodismo intercultural" (Browne y Pacheco, en Cuadernos de Información 29, pp. 133-140), "Comunicación intercultural mediada: construcción de realidad a través de un análisis crítico y complejo de los discursos periodísticos entre Chile y Perú" (Browne y Yáñez, en Revista Alpha 34, pp. 173-196) y "Análisis crítico del discurso de la representación intercultural en la prensa chilena" (Browne y Castillo-Hinojosa, en Convergencia: Revista de Ciencias Sociales 20(62), pp. 45-69). En este último los autores manifiestan que "las empresas periodísticas chilenas han sido protagonistas en la formación de la historia del país y en la construcción del Estado-Nación (...) los discursos periodísticos influyen en los procesos de construcción social de la realidad" (Browne y Castillo-Hinojosa 2013:47).

Dentro del enfoque comunicológico y de estudios mediales se encuentra la tesis doctoral de Alberto Mayorga defendida en el año 2013 en la Universidad Autónoma de Barcelona. En este trabajo titulado "Medios de comunicación, imaginarios sociales y poder. El discurso de la prensa chilena y argentina en torno a la acción colectiva de protesta y la crisis argentina de 2001", el autor se planteó como objetivo principal comprender el imaginario social de la acción colectiva de protesta y la crisis argentina de 2001 en el discurso editorial de los diarios El Mercurio de Chile y La Nación de Argentina. En el marco teóricometodológico de este trabajo, el autor recurre a los planteamientos de Baeza, Baczko, Berger, Castoriadis, Charaudeau, Luckmann, Pintos, Schutz, Van Dijk, entre otros. Esta investigación doctoral le permitió al autor desarrollar un programa de investigación en imaginarios sociales, discurso y poder, adscrito al Grupo de Investigación en Comunicación y Saberes Críticos de la Universidad de la Frontera.

Dentro de esta perspectiva se encuentran otros artículos que nacen del Grupo de Investigación en Comunicación y Saberes Críticos, en los que ha participado Luis Nitrihual y que ha publicado con el propio Mayorga, así como con otros investigadores tales como Carlos Del Valle, Rodrigo Browne, Juan Fierro, Macarena Herrera, Carolina Nahuelpi y Pedro Soto. Algunos de estos trabajos fueron desarrollos en el marco de un proyecto FONDECYT y apoyados por la Dirección de Investigación de la Universidad de la Frontera. En el marco de las directrices recién mencionadas se encuentra el artículo "El imaginario social de la mujer mapuche en el discurso de la prensa en Chile. El ejemplo del diario El Austral de la Araucanía" (Mayorga, Nahuelpi y Nitrihual, en Estudios sobre el Mensaje Periodístico 2(19), pp. 767-782), producto de los proyectos FONDECYT (№ 1120904) y DIUFRO (DI11-0006) y (DI13-0042).

También en la línea del análisis crítico del discurso, tanto a nivel de estudios mediales masivos, como en la intermediación hacia otras plataformas mediales, es reconocido el trabajo de Pablo Segovia Lacoste. En el año 2006 publica "Imaginarios sociales mapuches en la prensa" (en Enfoque Sociológico 6, pp. 18-30), donde desarrolla un análisis crítico del discurso emitido por la prensa chilena en distintos momentos históricos, constatando la configuración de un imaginario mapuche que menosprecia a este pueblo originario, desde la visión de parte de la cultura occidental chilena. Como dice el investigador, "esto nos 
remite necesariamente hacia las construcciones imaginarias, que los redactores de noticias vehiculan en los textos periodísticos sobre los mapuches, los chilenos y las relaciones que se construyen entre ellos en un determinado territorio" (Segovia 2006:28). El año 2013 es publicado otro estudio medial de Segovia, en co-autoría con Maritza Nieto, titulado "El contrato de comunicación en dos programas radiales chilenos" (en Revista Latinoamericana de Estudios del Discurso 2(13), pp. 99-120). En este trabajo se analiza los programas El gran tribunal de radio Pudahuel y El ranking musical de radio Penco, en la búsqueda de identificar cómo las interacciones radiofónicas locutor/radioescucha son un espacio discursivo donde se construyen y se refuerzan las identidades, mediante la construcción de un espectáculo discursivo que ficcionaliza una conversación íntima. Es decir, se hace referencia a cómo el juego de las imágenes o imaginarios que emanan a través del discurso construyen identidad local, proyectándose con fuerza a través del espacio radial.

Por otra parte, Rubén Dittus ha explorado exhaustivamente las dimensiones y alcances de los imaginarios sociales desde un diálogo con la semiótica. A través de la Tesis del Ojo Semiótico, un modelo de representación de la semiosis social que se trabaja desde corpus textuales con múltiples aplicaciones, Dittus explica que las imágenes psíquicas se encuentran en la forma de esquemas de representación o matrices de sentido, a través de los cuales se verían los mundos posibles, delimitando así lo visible/ invisible. En este enfoque el imaginario social es el punto ciego, desde y hacia la semiosis social. El Ojo Semiótico recupera la metáfora del lente y del enfoque semiótico: el observador semiotiza y es semiotizado por el campo cognoscitivo que estudia. En relación a los artículos científicos publicados por Dittus, destacamos "La opinión pública y los imaginarios sociales: hacia una redefinición de la espiral del silencio" (en Athenea Digital 7, pp. 61-76). En este trabajo, el autor manifiesta que la opinión pública no es más que un imaginario social y que los medios de comunicación de masas cumplen la función de ser recreadores simbólicos de la realidad. En tanto, en el ensayo "El imaginario social y su aporte a la teoría de la comunicación. Seis argumentos para debatir" (en Cinta de Moebio 26, pp. 166-176), el investigador presenta en seis argumentos las implicancias de la teoría de los imaginarios sociales en la comprensión del fenómeno comunicativo y su papel en el proceso de la semiosis. En esta reflexión y abordaje teórico, Dittus señala que "los imaginarios sociales son un factor de equilibrio psicosocial. Actúan compensando las diferencias y vacíos cognitivos, superando el excesivo racionalismo de la modernidad. De este modo, fortalecen la tendencia conservadora de todo orden social hacia su permanencia y reproducción" (Dittus 2006:173). Al momento de escribir este artículo, Dittus y un equipo de investigadores reunido en el núcleo "Guion Chile" (FONDECYT N¹160637 y N¹1130680) estudia la construcción del relato audiovisual para cine, televisión y transmedia y las metodologías asociadas a la percepción del perfil del guionista profesional y su imaginario como creador de historias y como articulador de representaciones en formatos audiovisuales. El texto "Fundamentos para un estudio del guion en el cine chileno: notas preliminares" (en Aisthesis 58, pp. 237-254) presenta las bases teóricas del estudio del guion y la figura del guionista, quien aprende de los principios básicos de la dramaturgia para construir universos simbólicos ficcionales. Estas se explican en manuales de escritura, y muchos de esos textos son usados por las escuelas y talleres de guion en todo el mundo. Recientemente, en una línea comunicativa más clásica, se explora la teoría de la autopoiesis de Humberto Maturana y sus alcances en la construcción de las subjetividades internas de una organización en el trabajo "Abriendo autopoiesis: implicancias para el estudio de la comunicación organizacional" (Dittus y Vásquez, en Cinta de Moebio 56, pp. 136-146). Una de las conclusiones de este trabajo indica que "cada observador lleva consigo toda una trama relacional, dentro de la cual el modo de pensar, hablar y actuar tiene sentido. Eso significa que un encuentro entre dos individuos es y será siempre personal, ambos inevitablemente forman parte de una dinámica sistémica que los vincula a relaciones simbólicas previas" (Dittus y Vásquez 2016:144). 
Por último, es necesario hacer referencia a los trabajos de Ignacio Riffo, quien investiga la perspectiva de los imaginarios sociales en la comunicación política, donde aborda principalmente las aportaciones de Cornelius Castoriadis, Bronislaw Baczko, Celso Sánchez, Manuel Baeza y, en mayor medida, el legado de Gilbert Durand y la escuela de Grenoble. Riffo se ha propuesto elaborar una propedéutica teórica para el estudio de los imaginarios mediáticos. De este trabajo se desprendieron algunas exposiciones en congresos, capítulos de libro y artículos científicos, como "Una reflexión para la comprensión de los imaginarios sociales" (en Revista Comuni@cción 7(1), pp. 63-76). El autor pretende analizar las estructuras imaginarias sociales presentes en los medios masivos de comunicación, poniendo de relieve a los imaginarios sociales como unas gramáticas estructurantes e instituyentes de las sociedades, destacando a la imaginación como la facultad creadora vital e irreductible del anthropos. Finalmente, también es meritorio indicar el artículo "La revitalización del héroe en las series norteamericanas. El caso de Doctor de House" (en Imagonautas 2(3), pp. 66-83), donde se planteó relacionar la figura del héroe con en el personaje protagónico de la serie Doctor House basándose especialmente en los fundamentos mitológicos de Joseph Campbell y Hugo Bauza.

\section{Reflexiones finales}

Los enfoques presentados ayudan a comprender el abordaje profundo al que se ven expuestos los fenómenos sociales y culturales del Chile simbólico contemporáneo. Los autores y teorías con las que se trabajan proponen un andamiaje conceptual de gran densidad y transversalidad. La dificultad que se observa es principalmente metodológica, ya que, si bien los textos canónicos de los teóricos del imaginario son conocidos y traducidos, no ocurre lo mismo con los trabajos dedicados a aspectos metodológicos. La excepción a esta tendencia son los trabajos del gallego Juan Luis Pintos, de gran influencia en algunos investigadores y ex discípulos chilenos, y Manuel Antonio Baeza, heredero de la escuela sociológica francesa, quienes han hecho un esfuerzo por abordar las dimensiones teóricas y metodológicas del imaginario.

Desde la mirada antropológica, coincidimos con la opinión de Belén Ortega, para quien la necesidad de encontrar un camino propio de investigación ha llevado a una operacionalización de la dimensión simbólica, que enmarca los elementos inmateriales de la praxis social, no solo de los imaginarios y las representaciones, sino también sus vehículos: imágenes, símbolos, actos, ritos o mitos. En ese sentido, la deuda pendiente para la comprensión de los problemas sociales es la correcta y útil inclusión lo simbólico. Esto permitiría -a juicio de Ortega- captar significados y sentidos de las necesidades y problemáticas vinculadas a los que las vivencian, sus futuros posibles, pero también los modelos y alternativas culturales y socio-políticos desde los cuales esas definiciones, categorizaciones y respuestas se formulan. Desde luego, creemos que incorporar lo simbólico a las problemáticas sociales en Chile ha permitido una visión holística de las dinámicas entre la sociedad y quienes viven en ella, pero la influencia de estos enfoques en las políticas públicas es, a lo menos, insuficiente. En palabras de la investigadora, una adecuada delimitación de los imaginarios para la investigación y la acción destinada al cambio se ve obstaculizada por la doble dimensión físico/virtual de las relaciones contemporáneas y las enacciones -en términos de Francisco Varela- como representaciones emergentes. Recordemos que según Varela no se puede entender el conocimiento sin sentido común, es decir, sin la capacidad que el observador tiene de observar su propia historia corporal y social, por lo tanto, el conocedor y conocido, sujeto y objeto, surgen simultáneamente.

Por otro lado, se constata un uso indiscriminado del término "representaciones", ligadas a los dispositivos de poder, sin que se haga distinción entre aquellas representaciones simbólicas (ligado a significados en 
sistemas de convivencia) y representaciones sociales (ligado a significados en estructuras sociales), aunque estén ambas conectadas. Del mismo modo, pareciera que el uso de "lo imaginario" está más asociado a la posibilidad de cambio y a lo discursivo (en su sentido más amplio). Lo anterior supondría que los imaginarios sociales se han convertido en una buena herramienta teórica para comprender la realidad, en un instrumento que ofrece un recorrido teórico y reflexivo importante, pues facilita preguntas de investigación. Sin embargo, pareciera que es insuficiente en sí mismo para interpretar enteramente el objeto de estudio, dada la dimensión holística que se requiere para su absoluta comprensión.

Otras dificultades se relacionan con la poca importancia que se da a la investigación en ciencias sociales en Chile y a los temas "no tangibles", lo que repercute en problemas de índole práctica: hay falta de oportunidades y financiamiento para desarrollar dichos temas, lo que finalmente afecta el resultado de las investigaciones realizadas. Este infausto escenario que pudimos identificar responde, en cierta media, a la iconoclasia endémica de Occidente que desvelaba Durand. Esta erosión de lo imaginario y el desplazamiento de la imaginación a las galeras del conocimiento se pueden comprender por el triunfo -a la fuerza- del positivismo en general y al historicismo y el cientificismo en particular como doctrina decimonónica que reconoce una "cosa" como verdadera, cuando esta "cosa" puede ser sometida a la instrumentalización del método científico. En este sentido, la imagen, lo imaginario y lo simbólico son señalados como oscuros, imprecisos, sospechosos e inductores al error, adjetivaciones que explican la escasa incorporación de dicho campo epistémico en un sistema que rinde culto a lo cuantitativo, a lo medible, a lo empírico y al progreso material.

\section{Nota}

Este artículo se enmarca en el proyecto de cooperación internacional titulado "Estado de la investigación en Iberoamérica en torno a los imaginarios y las representaciones sociales", que espera conformarse como un estado del arte en los diferentes países que se articulan (Argentina, Bolivia, Brasil, Colombia, Cuba, Chile, Ecuador, España, Honduras, México, Paraguay, Perú y Venezuela) a través de la Red Iberoamericana de Investigación en Imaginarios y Representaciones (RIIR). El proyecto cuenta con el aval académico de la Facultad de Sociología de la Universidad Santo Tomás (Colombia).

\section{Bibliografía}

Baeza, M.A. 2003. Imaginarios sociales. Apuntes para la discusión teórica y metodológica. Concepción: Ediciones Universidad de Concepción.

Baeza, M.A. 2008. Mundo real, mundo imaginario social. Teoría y práctica de sociología profunda. Santiago: RIL.

Baeza, M.A. 2016. Hacer mundo: significaciones imaginario-sociales para construir sociedad. Santiago: RIL.

Baeza, M.A. y Aravena, A. 2010. Jóvenes chilenos y construcción socio-imaginaria del ser-otro mujer. Última Década 32: 159-171.

Browne, R. y Castillo-Hinojosa, A. 2013. Análisis crítico del discurso de la representación intercultural en la prensa chilena. Convergencia: Revista de Ciencias Sociales 20(62): 45-69. http://ref.scielo.org/2wwfb4 
De la Garza Toledo, E. 2001. La epistemología crítica y el concepto de configuración. Revista Mexicana de Sociología 1: 109-127.

Dittus, R. 2006. El imaginario social y su aporte a la teoría de la comunicación: seis argumentos para debatir. Cinta moebio 26: 166-176. www.moebio.uchile.cl/26/dittus.htm

Dittus, R. y Vásquez, C. 2016. Abriendo la autopoiesis: implicancias para el estudio de la comunicación organizacional. Cinta moebio 56: 136-146. doi: 10.4067/S0717-554X2016000200002

Segovia, P. 2006. Imaginarios sociales mapuches en la prensa. Enfoque Sociológico 6: 18-30.

Recibido el 13 Dic 2016

Aceptado el 4 Feb 2017 